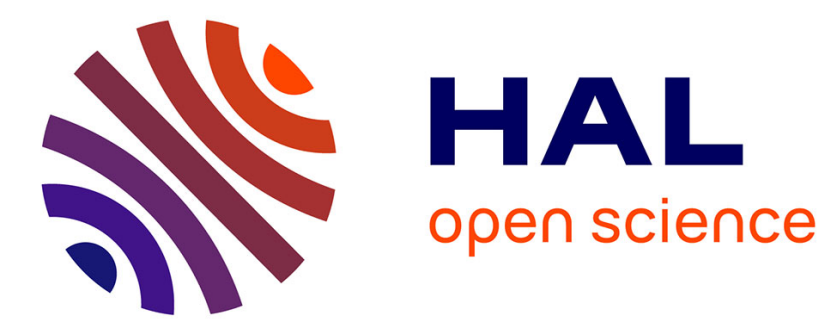

\title{
SMALL DROPS OF SATURATING FLUIDS
}

\section{J. Treiner}

\section{To cite this version:}

J. Treiner. SMALL DROPS OF SATURATING FLUIDS. International Workshop on Semiclassical and Phase Space Approaches to the Dynamics of the Nucleus, 1987, Aussois, France. pp.C2-107-C2116, 10.1051/jphyscol:1987217 . jpa-00226482

\section{HAL Id: jpa-00226482 https://hal.science/jpa-00226482}

Submitted on 1 Jan 1987

HAL is a multi-disciplinary open access archive for the deposit and dissemination of scientific research documents, whether they are published or not. The documents may come from teaching and research institutions in France or abroad, or from public or private research centers.
L'archive ouverte pluridisciplinaire HAL, est destinée au dépôt et à la diffusion de documents scientifiques de niveau recherche, publiés ou non, émanant des établissements d'enseignement et de recherche français ou étrangers, des laboratoires publics ou privés. 


\title{
SMALL DROPS OF SATURATING FLUIDS
}

\author{
J . TREINER \\ Division de Physique Théorique, Institut de Physique Nucléaire, \\ F-91406 orsay Cedex, France
}

\section{Abstract}

We present a semiclassical study of the breakdown of the leptodermous character of drops of quantum saturating fluids. A comparison is made between liquid ${ }^{4} \mathrm{He},{ }^{3} \mathrm{He}$ and nuclear matter. We discuss the size of the smallest bound drop in connection with the statistics obeyed by the particles and the structure of the bulk energy density. The existence of metastable fermionic systems with positive energy and negative chemical potential is also investigated.

\section{INTRODUCTION}

S. Stringari in his contribution to this meeting has outlined the similarities and specificities of different quantum fluids, namely nuclear matter, liquid ${ }^{3} \mathrm{He}$ and liquid ${ }^{4} \mathrm{He}$.

In the present contribution I would like to concentrate on one aspect of this discussion, which is concerned with the limit of very small systems bound by saturating forces. One of the question we will address is: what determines the size of the smallest bound drop? In the case of nuclei, one can go down to 2 particles ; in the case of helium, clusters have not yet been produced experimentally and we have to rely on their theoretical "production", which are discussed in refs.[1,2]; from these calculations, it appears that ${ }^{4} \mathrm{He}$ clusters are always bound whereas in the case of ${ }^{3} \mathrm{He}$, one needs at least $\simeq 30$ atoms to form a bound drop ; smaller clusters are found with positive energy and negative chemical potentials. Can we understand these features in simple terms?

A word about shell effects is here in order; as should be done in a meeting on semiclassical methods, I will distinguish between average trends and shell structure, except of course in the case of ${ }^{4} \mathrm{He}$. In the case of ${ }^{3} \mathrm{He}$, the results of the mean field calculations of ref.[2] indicate that the existence of shells manifests itself in a different way than in nuclei. On one hand the shell structure on the separation energies is relatively more pronounced than in nuclei, because i) the effective interaction can be considered purely central and $i i)$ the surface diffuseness is large (the ratio of the surface diffuseness to the mean separation distance is of the order of 1 in the case of ${ }^{3} \mathrm{He}$ whereas it is of the order of 0.5 in the case of nuclei); hence the degeneracy of the major shells resembles that of a pure harmonic oscillator. On the other hand, the existence of shells does not produce any oscillation of the density distributions, as is the case in mean field calculations of nuclei. This is due to several effects : to the large value of the effective mass $\left(m^{*} \simeq 3 m\right)$ which makes quantum effects to be less active; to the large surface energy ( $\simeq 3$ times 
the volume energy) and the large incompressibility ( $\simeq 40$ times the volume energy) which make the occurrence of density oscillations energetically unfavorable (in ref.[1], variational calculations were performed using correlated basis functions and a realistic atom-atom interaction; some density oscillations were indeed found in medium sized ${ }^{3} \mathrm{He}$ clusters and in small ${ }^{4}$ He clusters; however they seem to reflect the structure of the trial functions used in the minimization and should probably be considered as spurious).

The reduction of the quantum structure in ${ }^{3} \mathrm{He}$ clusters discussed above indicates that even in small clusters a semiclassical description will be appropriate. Actually it is shown in ref.[2] that the semiclassical density distributions of ${ }^{3} \mathrm{He}$ clusters coincide with the quantum mean field results.

\section{A DENSITY FUNCTIONAL APPROACH}

We assume that the energy of the system can be written as :

$$
E=\int \mathbb{H}\left(p, p^{\cdot} \ldots\right) \mathrm{d}^{3} r
$$

This assumption is morally supported by the Kohn-Horenberg theorem and practically worked out by using skyrme-type forces together with a semiclassical approximation for the kinetic energy density [3]. The structure of the functionals commonly used (consider one type of particles only) is the following :

$$
E=\int\left[F(\rho)+G(\rho)(\nabla \rho)^{2}\right] d^{3} x
$$

where $F(p) / p$ represents the bulk energy density and $G(p)$ characterizes the effective interaction in the surface region. $G(p)$ is usually positive for all values of $p$. The density $p(r)$ is determined by the Euler equation :

$$
\frac{d F}{d \rho}-\frac{d G}{d \rho} \rho^{\prime 2}-2 G \Delta \rho=\lambda
$$

where $\lambda$ is the chemical potential. Due to the saturating property, the energy can be expanded in powers of $A^{-1 / 3}$ ( $A$ is the number of particles)

$$
E=a_{v} A+a_{s} A^{2 / 3}+\ldots
$$

and the separation energy $\lambda$ is given by

$$
\lambda=\frac{\mathrm{dE}}{\mathrm{dA}}=\mathrm{a}_{\mathrm{v}}+\frac{2}{3} \mathrm{a}_{\mathrm{s}} \mathrm{A}^{-1 / 3}+\ldots
$$

Two examples of density distributions of helium clusters, taken from ref.[2], are shown in fig.(1), where are also given the results of ref.[1]. In the case of ${ }^{3} \mathrm{He}$, the quantum mean field curve and the semiclassical one are undistinguishable.

The saturating character of the interaction implies that the radius $R$ of the drop (which is spherical if one neglects shell effects), is proportional to $A^{1 / 3}$. The surface thickness $t$, which is of the order of the range of the force, is almost independent of 
$A$; hence the dimensionless parameter $t / R \simeq A^{-1 / 3}$ can be used as an expansion parameter characterizing the departure of any property of the finite drop from the corresponding property of the uniform medium. Such systems are known as "leptodermous systems". When considering smaller drops, the expansion in powers of $A^{-1 / 3}$ becomes questionable and indeed new terms, non-analytical in $A^{-1 / 3}$ arise ; the leptodermous regime goes into a "pachydermous" one and finally to a "holodermous" regime where the drop is pure surface.

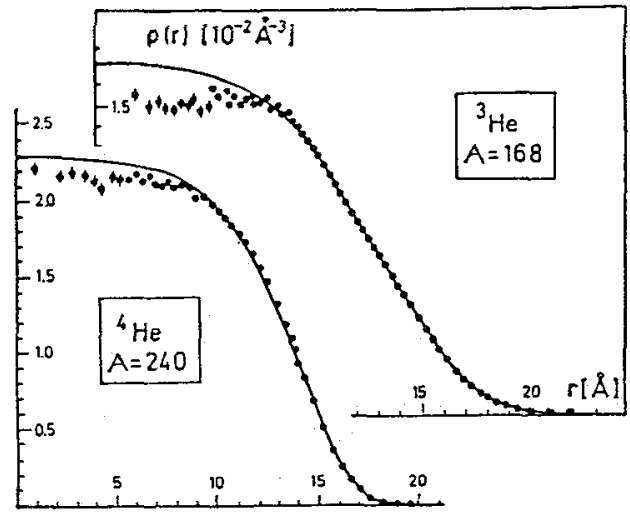

Eig.1 - Density distributions of two helium clusters. Straight line : mean field calculation of ref.[2] dots : exact calculations of ref.[1].

I shall in the following discuss two aspects of such systems which are, as we shall see, closely related :

i) a simple prediction of the Droplet Model which states that the bulk region of a drop should be compressed by the surface tension. ii) what determines the size of the smallest bound drop.

\section{COMPRESSION OF THE BULK BY THE SURFACE TENSION}

Let us first consider a simple prediction of the Droplet Model, stating that the central density of a finite drop should be larger than the saturation density $p_{0}$. The energy involved in compressing the bulk is proportional to $\mathrm{a}_{\mathrm{s}} \mathrm{A}^{2 / 3}$ and the energy involved in the resistance of the bulk is of the order $K A$, where $K$ is the incompressibility modulus. Hence the relative compression is proportional to the ratio of these two quantities. The exact result is :

$$
\frac{\delta p}{P_{0}} \equiv \frac{P_{c}-P_{0}}{P_{0}}=6 \frac{a_{s}}{K} A^{-1 / 3}
$$

where $p_{c}=p(r=0)$. However this prediction : "smaller drops should be more compressed", fails for small systems. On fig.(2) are reproduced the density distributions of He clusters of ref.[1]. The semiclassical results for hypothetical nuclei where the spin-orbit and the coulomb interaction have been suppressed are shown in fig. (3), together with the results concerning ${ }^{3} \mathrm{He}$ and ${ }^{4} \mathrm{He}$ clusters. In the case of nuclei, the prediction of eq.(1) is fulfilled for $A, 100$ in the case of SkM interaction and $A>40$ for 
SIII. For smaller drops, there is a "down-turn" of the curve and smaller drops are less compressed. For ${ }^{3} \mathrm{He}$ and ${ }^{4} \mathrm{He}$ clusters, the transition takes place around $A \simeq 300$ and 200 respectively.

This question has been discussed in ref. [4] and $I$ will just sketch here the argument. If, in the Euler equation, one neglects all derivatives at $r=0$, one gets :

$$
\frac{d F}{d p}=\lambda=a_{v}+\frac{2}{3} a_{s} A^{-1 / 3}+\ldots
$$

with

$$
\frac{d F}{d p}=\left.\frac{d F}{d p}\right|_{0}+\left.\frac{d^{2} F}{d p^{2}}\right|_{0} \delta p=a_{v}+\frac{k}{9 p_{0}} \delta p
$$

so one recovers eq. (6). One also obtains the result that if $\lambda$ is $a$ monotonic function of $A$, as it surely is, then $\delta p / p$ is also monotonic; in particular the down-turn cannot be obtained by considering more terms in the $\mathrm{A}^{-1 / 3}$ expansion of $\lambda$ (curvature or higher order terms), as was suggested in ref.[3]; the monotonic behaviour is linked to the assumption of zero derivatives at $r=0$.
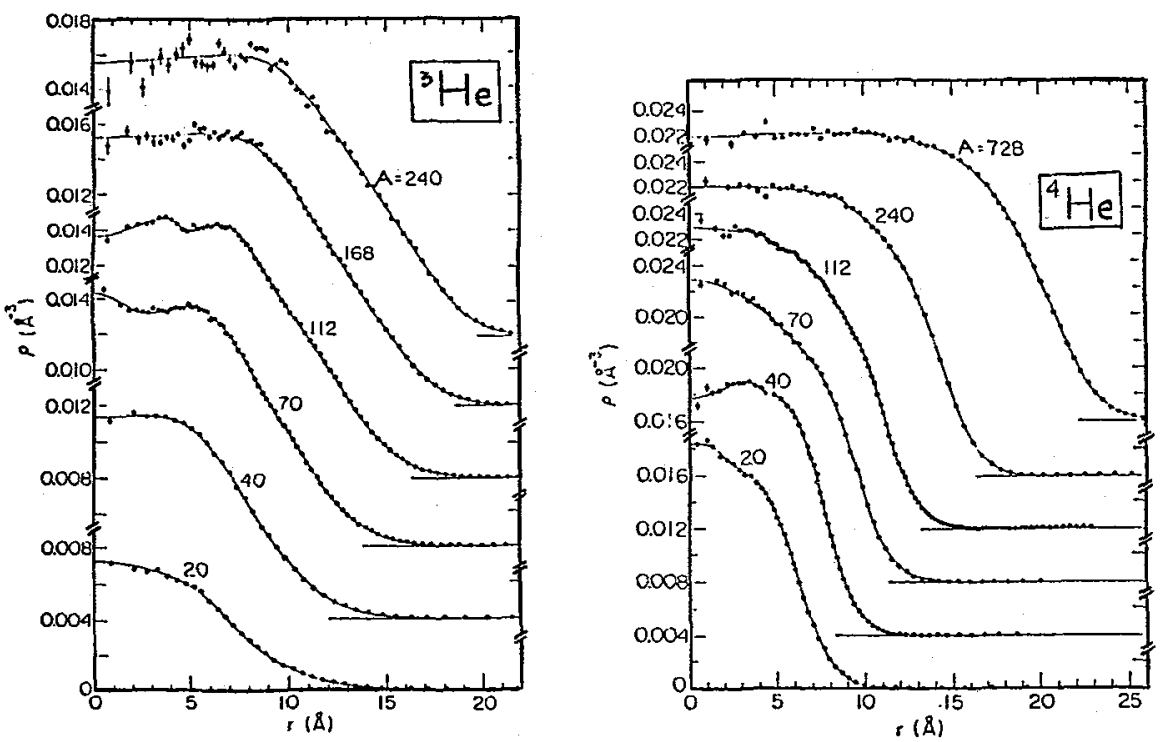

Fig.2 - Density distributions of helium clusters from ref.[1].

Indeed, while the first derivative of the density vanishes at the origin, the second derivative does not, and the Euler equation at $r=0$ actually reads

$$
\frac{d F}{d \rho}-2 G \Delta p=\lambda
$$

with $\Delta p(0)=3 p "(0)$. The equation replacing eq. (1) now reads

$$
\frac{\delta_{p}}{p_{0}}=6 \frac{a_{8}}{K} A^{-1 / 3}+3 \frac{a_{1 n}^{2}}{p_{0}} p(0)
$$


where $a_{1 \mathrm{n}}$ characterizes the exponential behaviour of the density away from the surface

$$
a_{i n} \simeq \sqrt{\frac{18}{K} G\left(p_{0}\right) p_{0}}
$$

and one also has

$$
p^{\prime \prime}(0) \alpha-\exp \left(-\frac{R}{a_{1 n}}\right)=-\exp \left(-\frac{r_{0}}{a_{1 n}} A^{1 / 3}\right)
$$
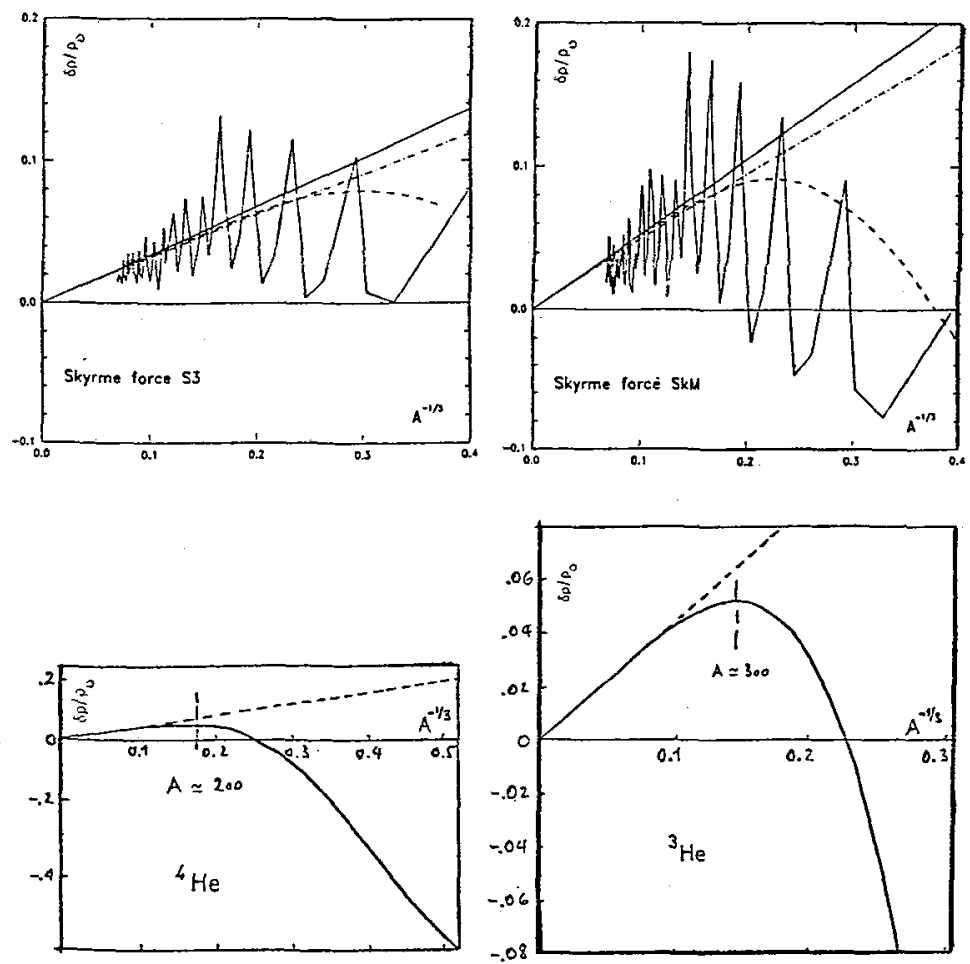

Fig.3 - Relative central compression as function of mass number. Upper curves from ref.[4]; broken curve : HF ; dashed line : semiclassical ; straight line : eq. (6); dashed dotted : eq. (6) plus curvature correction.

Lower curves from ref.[2]; full line : semiclassical ; dashed line: eq. (6).

Thus the down-turn in the relative compression is produced by this exponential term, which becomes dominant over the leptodermous one below a certain critical mass $A_{c}$. The presence of such an exponential term expresses the breakiown of the leptodermous behaviour of the drop, which becomes pachydermous. The magnitude of this term is governed by the ratio of the mean distance between the 
particles to the inward diffuseness. One should notice that this inward diffuseness may be significantly different from the global diffuseness of the surface profile, related to the total surface thickness ; for example for semi-infinite nuclear matter, one finds [5] that $a_{i n} \simeq 0.8-0.9 \mathrm{fm}$ (for $\mathrm{K}=200 \mathrm{MeV}$ ) whereas the global diffuseness is $\simeq 0.55 \mathrm{fm}$. In the case of liquid helium, one finds [6] that the surface profile is highly unsymmetrical and that the density decreases outward more rapidly than inward, so that most of the surface thickness is governed by $a_{1 a}$. Numerically one finds

$$
\begin{aligned}
& \frac{r_{0}}{a_{1 n}} \simeq 1.4 \text { for nuclear matter } \\
& \frac{r_{0}}{a_{1 n}} \simeq 1.1 \text { for } \mathrm{He} \\
& \frac{r_{0}}{a_{i n}} \simeq 1 \text { for }{ }^{3} \mathrm{He}
\end{aligned}
$$

From eq.(12), the size of the system where the transition from the leptodermous behavioux to the pachydermous one takes place is expected to vary as the cube of the ratio $a_{1 n} / x_{0}$. This expectation agrees with the values $A_{c} \simeq 100,200$ and 300 found respectively in fig. (2).

\section{SIZE OF THE SMALLEST BOUND DROP}

We now turn to our second point. Let us start with the expression of the total energy of the drop

$$
E=\int\left[F(p)+G(p)\left(v_{p}\right)^{2}\right] d^{3} r
$$

The contribution of the gradient term is positive; the binding comes from the integral of $F(p)$. The function $F(p) / p$ for a uniform system of fermions is sketched in fig.(4); at low densities it is positive because the kinetic energy is dominant over the potential energy ; with increasing density the attraction is more effective and $F(p) / p$ becomes negative for a certain value

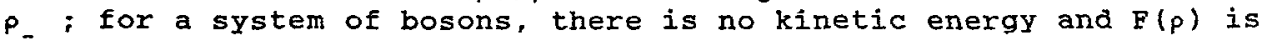
negative at low densities.

When considering the integral of $F$, we shall distinguish between $r<r$ and $r_{-}$, where $r$ represents the radius where the density is equal to $p_{-}^{-}$:

$$
F(p) d^{3} r=4 \pi \int_{0}^{\infty} r^{2} F(p) d r+4 \pi \int_{0}^{\infty} r^{2} F(p) d r
$$

The first term in the $r, h, s$ of eq. (13) is negative and the second one is positive. Hence for a cluster to be bound, the central density must be large enough so that the first term compensates for the second one and for the contribution of the gradient component. clearly, the smallest bound cluster must have $P$. significantly larger than $P_{-}$. Now we have seen above the central density of a 


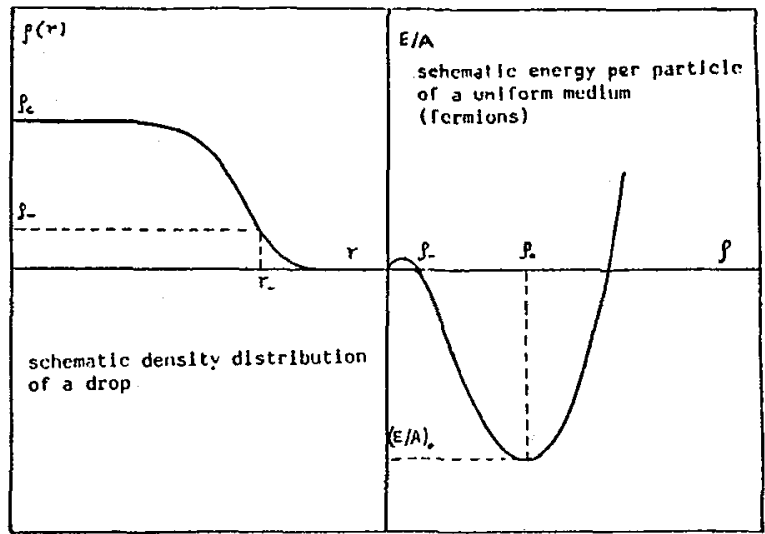

Fig.4

pachydermous drop decreases with the number of particles ; therefore for a system of fermions one necessarily reaches a finite limit to the size of the smallest bound drop. The smaller the value of $P_{-}$, the smaller the size of this drop. For a system of bosons it is not so, because in order to minimize the energy, the system will develop a smooth surface with small gradients; consequently the central density will be small, but the integral of F will still be negative. One thus expects that any number of bosons will form a bound system.

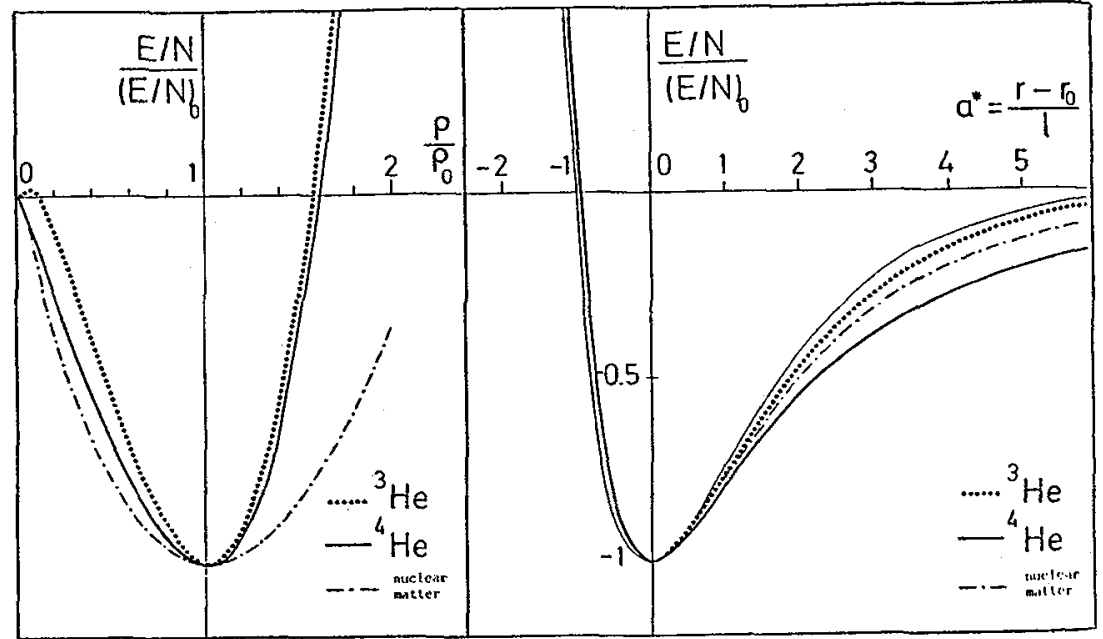

Fig.5 - a : binding energy versus density in dimensionless coordinates

b : same as a using the abscissa defined in eq. (19); thin line from ref.[8].

The actual curves for the three quantum liquids considered here are shown in fig. (5a), using dimensionless coordinates. One finds the following results : 


$$
\begin{aligned}
& \frac{P_{-}}{P_{0}}=0 \text { for }{ }^{4} \mathrm{He} \\
& \frac{P_{-}}{P_{0}}=0.15 \text { for }{ }^{3} \mathrm{He} \text { and } \\
& \frac{P_{-}}{P_{0}} \simeq 10^{-3} \text { for nuclear matter. }
\end{aligned}
$$

These values explain why the number of particles $\mathbf{A}_{\ell}$ of the smallest bound ${ }^{3}$ He cluster is appreciable ( $\simeq 30$ atoms) while in the case of nuclei one can go down to 2 particles.

Notice that it is not the value of the surface energy which governs the value of $A_{\ell}$. The surface energy depends on both functions $F(p)$ and $G(p)$. A depends only on $F(p)$.

A few years ago, Grammaticos discussed the same subject [7] and arrived at the conclusion that $A_{f}$ should be zero. However his result was obtained using a schematic functions which is attractive at all densities. Such a functional, as we have seen, is not appropriate for the discussion of fermionic systems.

\section{v. METASTABLE DROPS OF FERMIONS}

It is possible to show, from the Euler equation, that for the smallest bound drop, whose binding energy $E_{\ell}$ is zero, the chemical potential is strictly negative. One multiplies eq. (3) by $p^{\prime}$ and integrate between $+\infty$ and some radius $r$; the result is

$$
F(p)-\int_{\infty}^{r} \frac{d r}{r^{4}} \frac{d}{d r}\left[r^{4} G(p) p^{2}\right]=\lambda p
$$

which, after integrating by parts, can be written as

$$
F(p)-G(p) p^{2}-4 \int_{\infty}^{r} G(p) \frac{p^{2}}{r} d r=\lambda \rho
$$

We now integrate eq.(15) over all space; after permuting the order of integrations in the last term of the r.h.s. one gets

$$
\int\left[F(p)-G(p) p^{\prime 2}\right] d^{3} r+\frac{4}{3} \int G(p) p^{\prime 2} d^{3} r=\lambda A
$$

Now the smallest drop has zero binding energy, i.e.

$$
\int\left[F(p)+G(p) p^{\prime 2}\right] d^{3} r=0
$$

Introducing eq. (17) into eq.(16) leads to

$$
\lambda_{\ell}=-\frac{2}{3 A_{\ell}} \int G(p) p^{\prime 2} d^{3} r
$$

which is a negative quantity. Moreover one has necessarily $\lambda<E / A$, because the energy per particle is a decreasing function of the 
number of particles. Thus one can find numerical solutions of the Euler equation for $A<A$, until the chemical potential reaches zero. ; this happens for a certain critical number of particles $A_{0}$. For $A_{0}<A<A_{\ell}$, one has metastable drops with positive energy and negative chemical potentials. The situation is schematically shown in $\mathrm{fig} .6$.

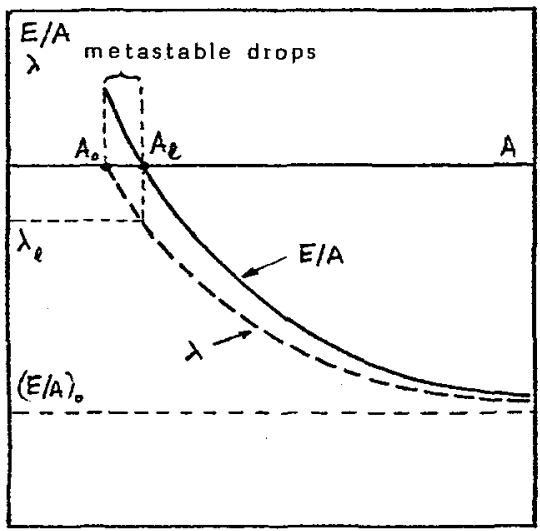

Fig.6 - Schematic representation of $E / A$ and $\lambda$ for small fermionic drops.

VI. UNIVERSAL BINDING-ENERGY-DISTANCE RELATION ?

We shall finish with a brief discussion of a recent work where arguments are given that the binding-energy-distance (or density) relation is universal. In order to exhibit this universality, the authors of ref.[8] define a scaled abscissa $a^{*}$ by

$$
a^{*}=\frac{r-r_{0}}{\ell}
$$

where $r$ is the inter-particle distance corresponding to the density $p$ and $\ell$ a length scale defined as

$$
e=r_{0} \sqrt{\frac{(E / A)_{0}}{R}}
$$

The resulting curves are plotted in fig.(5b); we also show for comparison the curve presented in fig.(1) of ref.[8], representing the binding-energy-distance relation for molecular and metallic systems. Although at short distance the scaling accounts satisfactorily for the small differences seen above saturation density in fig.(5a), the effect of the different statistics obeyed by liquid ${ }^{3} \mathrm{He}$ and ${ }^{4} \mathrm{He}$ shows up clearly for $\mathrm{a}^{*}>0$. The scaling length $\ell$ takes into account compressibility effects, however the relative variation of $K$ with density (related to the Grüneisen constant) may also by quite different for various systems; in particular it is relatively much larger in ${ }^{3}$ he than in nuclear matter. This explains why the curves for fermionic systems do not coincide in fig.(5b). 
The value of the ratio of surface to volume energy is partly related to the same question. The value proposed in ref.[8] ( $\simeq$ 0.82 ) does not apply for liquid helium quantitatively. This ratio is $\simeq 2.4$ for ${ }^{4} \mathrm{He}$ and $\simeq 3.4$ for ${ }^{3} \mathrm{He}$ (it is 1.2 for atomic nuclei). Relations between incompressibility, saturation density, surface tension and surface thickness have been investigated in particular in $\operatorname{refs} .[5,7,9]$.

\section{CONCLUSION}

We have tried in the present study to illustrate how rather detailed features of exact calculations can be understood in simple terms within a density functional approach. Concerning the compression of the bulk by the surface tension, we have seen that the parameter $\gamma$ governing the transition from the leptodermous regime where "smaller drops are more compressed" to the pachydermous regime where the opposite is true, is just the ratio of the inter-particle distance to the inward surface diffuseness of the surface. This parameter appears in an exponential term of the form $\exp \left(-\gamma^{1 / 3}\right)$. The size of the smallest bound drop is different for boson systems and for fermion systems. For bosons, any number of particles are able to form a bound drop. For fermions, the size of the smallest bound drop is related to the balance between the kinetic energy and the potential energy at low density, and not to the value of the surface energy, as one might have expected. For liquid ${ }^{3} \mathrm{He}$, the interaction is not strong enough and one needs at least $\simeq 30$ atoms to form a bound cluster. Below this mass, metastable drops with positive energy and negative chemical potential may exist. The absolute limit, corresponding to zero chemical potential, is found to be around 16 to 18 atoms.

This work is part of collaborations with $S$. Stringari and W.J. Swiatecki. It has also benefitted fruitful discussions with W.D. Myers and H. Krivine.

\section{References}

[1] V.R. Pandharipande, S.C. Pieper and R.B. Wiringa, Phys.Rev. B34 (1986) 4571 .

[2] S. Stringari and J. Treiner, to be published in J. Low Temp. Phys.

[3] M. Brack, C. Guet and H.B. Hakansson, Phys. Rep. 123 (1984) 276.

[4] J. Treiner, W.D. Myers, W.J. Swiatecki and M. Weiss, Nucl. Phys. A452 (1986) 93.

[5] J. Treiner and H. Krivine, Ann. Of Phys. 170 (1986) 406.

[6] S. Stringari and J. Treiner, to be published in Phys. Rev.B.

[7] B. Grammaticos, Workshop on Semiclassical Methods in Nuclear Physics, ILL-Grenoble, March 1981 ; Ann. of Phys. 139 (1982) 1 .

[8] J.H. Rose, J.P. Vary and J.R. Smith, Phys. Rev. B53 (1984) 344 and references therein.

[9] R.C. Brown and March, J. Phys. C6 (1973) L363;

$\mathrm{X}$. Campi and S. Stringari, Nucl. Phys. A337 (1980) 313. 